\title{
AN OVERVIEW OF DATASETS ON SMALL BODIES AVAILABLE THROUGH THE PLANETARY DATA SYSTEM AND SOARD
}

\author{
DONALD R. DAVIS \\ Planetary Science Institute, \\ 620 N. 6th Avenue, Tucson, Arizona 85705 \\ E-mail drd@psi.edu \\ MICHAEL A'HEARN \\ University of Maryland, College Park, Maryland 20742 \\ $E$-mail ma@astro.umd.edu \\ EDWIN GRAYZECK \\ University of Maryland, College Park, Maryland 20142 \\ E-mail grayzeck@stars.dnet.nasa.gov \\ MARK V. SYKES \\ University of Arizona, Tucson, Arizona 87521 \\ E-mail grayzeck@stars.dnet.nasa.gov \\ E.M. ALVAREZ DEL CASTILLO \\ National Optical Astronomy Observatories, \\ 950 North Cherry, Tucson, Arizona 85726 \\ E-mail ealvarez@noao.edu \\ DAVID THOLEN \\ Institute for Astronomy, \\ 2680 Woodlawn Drive, Honolulu, Hawaii 96822 \\ E-mail tholen@hale.ifa.hawaii.edu \\ KEVIN GARLOW \\ Steward Observatory, \\ University of Arizona, Tucson, Arizona 85721 \\ E-mail garlow@as.arizona.edu
}

\begin{abstract}
In this chapter we present an overview of NASA's Planetary Data System project with emphasis on the Small Bodies Node (SBN) and describe a separate relational database project, the Steward Observatory Asteroid Relational Database (SOARD). We summarize the datasets that are currently available through SBN and SOARD as well as those planned to be ingested in the future. Procedures for accessing data from the SBN and SOARD via Internet are included.
\end{abstract}




\section{Planetary Data System Overview}

The mission of the Planetary Data System (PDS) is to provide the scientific community electronic access to planetary spacecraft data and groundbased data whose science content has been reviewed and verified, to prepare datasets for long-term curation, and to provide knowledgeable scientific expertise to the user community in order to enhance and maximize the efficiency of data identification and access. Eventually, a user should be able to login to the PDS, identify, examine, extract or order a subset of the available data, request special products, and be provided with scientific and technical support. PDS also distributes datasets and provides user support so far as resources permit.

PDS comprises the following elements (Table 1): The Central Node, seven Discipline Nodes, and a Support Node, the Data Distribution Laboratory (DDL). One institution serves as the principal site for each node, and one scientist at each Discipline Node serves as that node manager.

\begin{tabular}{ll}
\hline \multicolumn{1}{c}{ Table 1 } \\
\hline \multicolumn{1}{c}{ NODE NAME } & \multicolumn{1}{c}{ LOCATION } \\
\hline $\begin{array}{l}\text { Central Node } \\
\text { Discipline Nodes } \\
\text { Geosciences }\end{array}$ & JPL \\
Atmospheres & Washington University \\
Plasma Interactions & University of Colorado \\
Small Bodies & UCLA \\
Imaging & University of Maryland \\
Planetary Rings & USGS - Flagstaff \\
Navigation and Ancillary Information Facility & JPL \\
Support Node: Data Distribution Lab & JPL \\
\hline \hline
\end{tabular}

The roles of the various nodes are as follows :

\section{CENTRAL NODE}

- Responsible for maintaining a high-level catalog of all datasets in the PDS, and interface software allowing the user to browse through this catalog and be pointed to the appropriate Discipline Node (eventually being seamlessly connected to that Discipline Node).

- The Central Node does not maintain any datasets, but may process orders for datasets made through them by passing those orders along to NSSDC or the Discipline Node. The CN is responsible, in consultation with the Discipline Nodes, for the development of standards for dataset content and format. They are charged with ensuring that those standards are maintained in the data ingestion process across all Discipline Nodes. 
- Responsible for maintaining standards and formats of distributed data sets on CDROMs across all Discipline Nodes.

DISCIPLINE NODES (DN)

- Responsible for the identification and ingestion of datasets pertaining to their subject area.

- Responsible for verification of dataset scientific content and quality.

- Responsible for interfacing with science teams of ongoing missions to design and archive data products consistent with PDS standards and then accomplish the ingestion of that data, thereby giving the science community widespread access to the data.

- Responsible for maintaining a copy of the above datasets which are to be made available online as practical with available equipment.

- Responsible for the development of their own online interface software allowing the users to examine in some detail the datasets for which the Discipline Node is responsible.

- Provide scientific expertise and human interaction to the user helping them to navigate and understand the available data. Respond to user requests insofar as resources allow for special processing of datasets suited for their research needs and data subsets automatically, without human intervention on the part of the Node.

- Responsible, in coordination and collaboration with the CN and DDL, for the production and large-scale distribution of mission datasets (usually undertaken by the missions themselves).

DATA DISTRIBUTION LABORATORY (DDL)

- Responsible for incorporating into the Discipline Nodes advanced technology for data archiving coming from work funded independently of PDS.

- Support DN scientists in the production and distribution of datasets using CDROMs.

\section{PDS Small Bodies Node Overview}

The Small Bodies Node (SBN) of the Planetary Data System (PDS) provides expertise on comets, asteroids, and interplanetary dust. The main node, located at the University of Maryland, College Park, acts as the primary contact point for the community. It processes requests for its holdings, acquires and archives new datasets, coordinates peer reviews of the archives, and provides software support for browsing the data archives and working with the archived dataset. The main node also provides scientific expertise on the cometary datasets. Two other subnodes, the Interplanetary Dust node at the University of Arizona, and the Asteroids node at the University of Hawaii and at the Planetary Science Institute in Tucson, provide scientific expertise in their respective fields to the user community as well as assisting in the collecting and archiving tasks of the SBN. An additional subnode at Konkoly Observatory, Budapest, Hungary, handles distribution of SBN holdings to the European scientific community and assists in collecting and archiving data from selected space missions in Europe. The current status of SBN datasets is summarized in Table 2. A more detailed listing of the current holdings (e.g., file size, etc.) and planned additions to the SBN may be obtained from E. Grayzeck. 
Current projects at the SBN include development of a Windows-based interface into the node data holdings which will provide catalog searching and some data browsing capabilities, integration of the NAIF software into the node interface to provide ephemeris calculations for comets and asteroids, and inclusion of the Steward Observatory Asteroid Relational Database (SOARD) in the SBN node interface.

\begin{tabular}{|c|c|c|c|c|}
\hline & \multicolumn{2}{|c|}{ Reviewed, Ingested and Available } & $\begin{array}{l}\text { Being Revised } \\
\text { Following Review }\end{array}$ & $\begin{array}{l}\text { Collected But Not } \\
\text { Reviewed }\end{array}$ \\
\hline Asteroid Data & \multicolumn{2}{|c|}{$\begin{array}{l}\text { Pole positions } \\
24 \text {-color } \\
\text { Asteroid name file }\end{array}$} & $\begin{array}{l}\text { Eight-color } \\
\text { Taxonomy } \\
\text { Magnitude and slope } \\
\text { parameters } \\
\text { (note that these } \\
\text { include many ancillary } \\
\text { files like the filter } \\
\text { transmission curves for } \\
\text { the ECAS filters) }\end{array}$ & $\begin{array}{l}\text { Cunningham } \\
\text { bibliography } \\
\text { Beginnings of a } \\
\text { lightcurve database } \\
\text { Zappalà's families } \\
\text { File of proper elements } \\
\text { Ostro's radar results } \\
\text { IRAS diameters }\end{array}$ \\
\hline Comet Data & \multicolumn{3}{|c|}{$\begin{array}{l}\text { IHW Earth-based - } \\
34 \text { datasets }\end{array}$} & $\begin{array}{l}\text { IHW Space Based - } \\
\text { Giotto } \\
\text { IHW Space based - } \\
\text { Vega } \\
\text { IHW Space based - } \\
\text { Suisea/Sakigaka/Ice }\end{array}$ \\
\hline Dust Data & IRAS & $\begin{array}{l}\text { Filters } \\
\text { Scan history } \\
\text { Med. Res. ZOHF } \\
\text { Low Resolution ZOHF }\end{array}$ & & Super Sky Flux \\
\hline
\end{tabular}

\section{An Interactive Database for Asteroid Data : SOARD}

Two of us (MS and EAdC) saw the need to have a tool for answering scientific questions using large datasets. Supported, in part, by the Space Engineering Resource Center (SERC) at the University of Arizona, they developed SOARD, a menu-driven database management system. This program uses relational database software (dBase IV) in a fashion transparent to the user to provide interactive and manipulative tools for accessing a variety of datasets. The initial development phase of SOARD is now complete and future improvements to it are supported jointly by the Asteroids subnode of the SBN and SERC. The datasets currently available in SOARD and the principal contributing scientists for the dataset are listed in Table 3.

Currently, SOARD has more asteroid datasets than does SBN; however, the SOARD datasets have not been peer-reviewed. It is our plan to replace the unreviewed datasets with the peer-reviewed versions for PDS/SBN as they become available. Thus, over time, the datasets within the two projects will become identical. We also plan to make SOARD accessible via the SBN interface, once that interface has been developed. 


\begin{tabular}{ll}
\hline \hline & \multicolumn{1}{c}{ Table 3 } \\
\hline Orbital Elements: & $\begin{array}{l}\text { Osculating Orbital Elements }{ }^{1} \text { (E. Bowell, MPC) } \\
\text { Proper Orbital Elements (Milani, Williams) }\end{array}$ \\
Classification Schemes: & Taxonomic Classifications (Tholen, Barucci, Tedesco, CMZ) \\
& Family Designations (Williams, Zappala) \\
Physical Parameter Datasets: & Magnitudes, Albedos, and Diameters (Tholen, IRAS) \\
& Photometric Parameters (MPC) \\
& Pole Orientations (Magnusson) \\
& Radar Albedos and Cross-sections (Ostro) \\
& Individual IRAS Observations (IRAS.FPND) \\
Observational Data: & Polarimetry data (IRAS.FPND) \\
& Lightcurve data (IRAS.FPND) \\
& UBV Observations (IRAS.FPND) \\
& 8-Color Spectroscopy (D. Tholen) \\
& 24-Color Spectroscopy (C. Chapman and M. Gaffey) \\
& 52-Color Spectroscopy (J. Bell) \\
& 3 Micron Spectroscopy (T. Jones) \\
& Asteroid Bibliography (C. Cunningham) \\
& Preliminary Designations and Discovery Circumstances \\
& (MPC) \\
\hline \hline
\end{tabular}

'This file contains orbits for more than 21,000 asteroids with an osculation date of 1993/04/23. This file will be updated on approximately a quarterly basis; see the chapter by $\mathbf{E}$. Bowell for additional information on osculating orbits.

SOARD allows users to : (1) browse through datasets to view parameters as well as the actual data, (2) define and extract subsets of database parameters for subsets of asteroids, (3) output files (tailored for plotting) of multi-band photometry or spectroscopy for individual asteroids, and (4) search for bibliographic references satisfying users' search criteria. After using SOARD to create the desired dataset, the user creates ASCII output to be "ftp'ed" back to the home computer. In addition, users may download the original datasets in ASCII format without using SOARD to extract subsets of data.

We are interested in collecting other datasets relating to comets, meteors and meteorites that could be incorporated into SOARD. If you have datasets to contribute, please contact Dr. Donald R. Davis or Dr. Mark V. Sykes at the appropriate address listed below.

\section{Accessing the Datasets and Future Additions}

Information for accessing the data sources described above via telnet is given in Table 4. Also listed in this Table are the scientific and system support personnel for each data source.

We anticipate further development in both SBN and SOARD capabilities, both through acquisition of additional datasets and by updates of existing ones. Many of the datasets are continuously updated and we plan to periodically update the various available datasets through both the SBN and SOARD. Information about the current version of a dataset will be supplied on-line. 


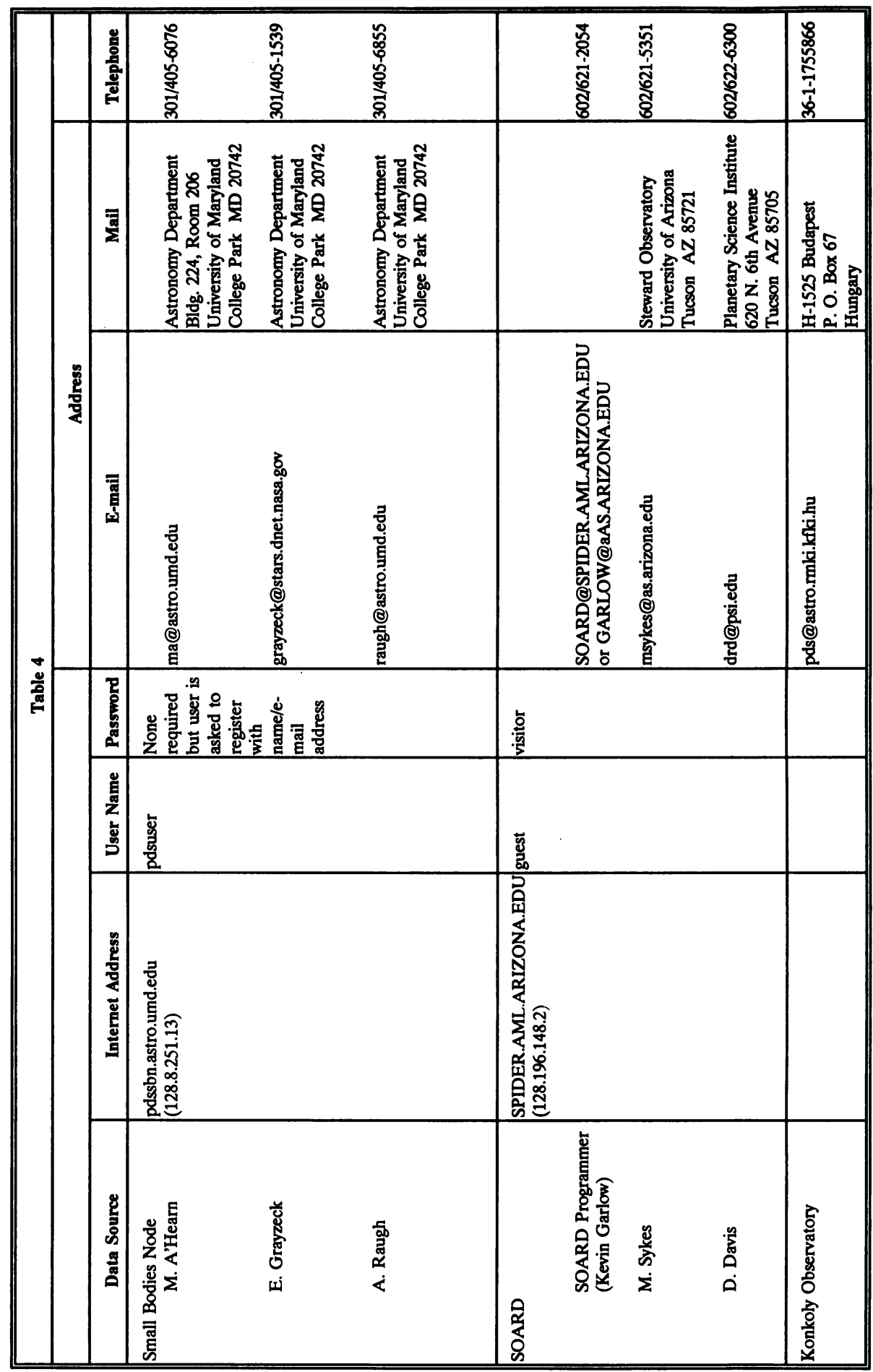

